\title{
PROYECCIONES DIDÁCTICAS DE LA TEORÍA
}

\author{
ARGUMENTATIVA DE LA RAZÓN
}

\section{Didactical projections \\ of the argumentative theory of reasoning}

\author{
RODRIGO SEBASTIÁN BRAICOVICH ${ }^{*}$ \\ Investigador independiente, CONICET, Rosario, Argentina \\ rbraicovich@gmail.com \\ Código Orcid: https://orcid.org/0000-0003-2785-7293
}

\begin{abstract}
Resumen
El objetivo del artículo consiste en poner en diálogo tres líneas de investigación dentro de la epistemología contemporánea:la Epistemología de la Virtud, el paradigma de la Racionalidad Limitada y la Teoría Argumentativa de la Razón. Frente al problema que interesa analizar aquí, a saber, la búsqueda de un marco teórico que permita diseñar estrategias pedagógicas (tanto al interior de la enseñanza de la filosofía como fuera de ella) sobre premisas realistas, la Epistemología de la Virtud será presentada como una corriente marcadamente optimista desde el punto de vista epistémico. El paradigma de la Racionalidad Limitada representará la contrapartida de dicha corriente, en la medida en que parece conducir a un pesimismo marcado respecto de la posibilidad de diseñar estrategias que permitan perfeccionar las prácticas epistémicas de los sujetos. Frente a estos dos polos, se sugerirá que la Teoría Argumentativa de la Razón (desarrollada en la última década por Hugo Mercier y Dan Sperber) representa una alternativa prometedora por dos razones fundamentales: en primer lugar, porque ofrece una respuesta al problema (enfrentado por el paradigma de la Racionalidad Limitada) del carácter adaptativo de la razón humana desde un punto de vista evolutivo; en segundo lugar, porque permite superar el pesimismo epistémico esencial al paradigma de la Racionalidad Limitada al momento de planificar estrategias pedagógicas realistas y efectivas.
\end{abstract}

Argumentación, racionalidad, crítica, limitada, epistemología, virtud, evolución.

Forma sugerida de citar: Braicovich, Rodrigo (2021). Proyecciones didácticas de la teoría argumentativa de la razón. Sophia, colección de Filosofía de la Educación, 30, pp. 203-223.

* Doctor en Humanidades y Artes con mención en Filosofía. Ha publicado artículos sobre cognitivismo y psicología de la acción desde la perspectiva de la antigüedad clásica y desde el pensamiento contemporáneo. La presente investigación cuenta con el apoyo y soporte financiero del Consejo Nacional de Investigaciones Científicas y Técnicas (CONICET) de Rosario, Santa Fe, Argentina. 


\begin{abstract}
The goal of the article aims at establishing a dialogue between three lines of inquiry within contemporary epistemology: Virtue Epistemology, Bounded Rationality and Argumentative Theory of Reasoning. Faced with the problem that we are interested in dealing with here, i.e., the search for a theoretical framework that might allow us to design pedagogic strategies (both within the framework of the didactic of philosophy and outside of it) based on realistic premises, Virtue Epistemology will be presented here as a strongly optimistic current from an epistemic viewpoint. The paradigm of Bounded Rationality will represent the exact counterpart, insofar as it seems to lead to a pronounced pessimism concerning the possibility of designing strategies that may allow us to improve the agent's epistemic practices. In the middle of these two extremes, the Argumentative Theory of Reasoning (developed in the last decade by Hugo Mercier and Dan Sperber) represents a promising alternative for two reasons: in the first place, because it offers an answer to the problem (faced by the paradigm of Bounded Rationality) of the adaptive character of human reason from an evolutionary viewpoint; secondly, because it allows us to overcome the epistemic pessimism that is essential to the paradigm of Bounded Rationality when planning pedagogical strategies that are not only realistic but also effective.
\end{abstract}

Keywords

Argumentation, rationality, critic, bounds, virtue, epistemology, evolution.

\title{
Introducción
}

La revolución cognitiva que tuvo lugar a mediados y fines del siglo pasado obligó a una revisión sustancial de la concepción clásica de la racionalidad humana, es decir, de la concepción de la misma que Occidente había heredado del pensamiento clásico y que había encontrado su expresión paradigmática, en lo que concierne al optimismo epistemológico, en el pensamiento de la Ilustración. El paradigma de la Racionalidad Limitada, en particular, contribuyó a un cuestionamiento decisivo de la confianza tradicional en la esencial perfectibilidad de la especie humana a partir de un desarrollo racional (tanto individual como colectivo) que operaría como clave del progreso humano. Los estudios iniciales de Herbert Simon respecto de los límites epistémicos inherentes a los procesos de decisión humanos, junto con el estudio sistemático de los sesgos cognitivos, condujeron, en efecto, a un panorama esencialmente desolador en cuanto a la confianza en la capacidad humana de instanciar mecanismos decisorios eficientes y racionales. Frente a tal escenario, la Teoría de la Argumentación Racional, desarrollada en la última década por Hugo Mercier y Dan Sperber, aparece como una alternativa innovadora, en la medida en que permite una relectura de las supuestas limitaciones de la racionalidad a partir de la reinserción de la arquitectura cognitiva humana en su escenario evolutivo original. Como se intentará mostrar, aun cuando dicho abordaje no avala la reinstauración del exacerbado optimismo en la racionalidad humana propio de la concepción clásica, permite al menos 
escapar a los derroteros marcadamente pesimistas del paradigma de la Racionalidad Limitada, al tiempo que abre vías de exploración y diseño de estrategias didácticas realistas y efectivas desde el punto de vista de la argumentación.

La exposición estará articulada de la siguiente forma: en la primera sección se expondrá el optimismo que caracteriza en forma esencial y explícita a la vertiente más importante y desarrollada de la Epistemología de la Virtud, mostrando cómo la misma representa una continuidad respecto del paradigma clásico de la racionalidad (tanto antiguo como Ilustrado). La segunda sección abordará el paradigma de la Racionalidad Limitada como un contrapunto exacto al optimismo propio del paradigma clásico, en la medida en que aspira a poner de relieve las limitaciones estructurales de la arquitectura cognitiva humana, ya sea a través del estudio de los límites en la computabilidad de los problemas que enfrentan los seres humanos en los escenarios de decisión, como a través de las investigaciones relativas a los sesgos cognitivos que atraviesan a la razón humana. Frente a ambos extremos, la tercera sección intentará mostrar que la Teoría Argumentativa de la Razón, aun cuando parte del paradigma de la Racionalidad Limitada, ofrece una alternativa novedosa frente a tales extremos, en tanto permite entender el surgimiento de la Razón humana a la luz de un paisaje evolutivo que explica la función central que asume la persuasión en el funcionamiento de la misma. La cuarta sección aborda las razones por las cuales la Teoría Argumentativa de la Razón permite reinterpretar los sesgos cognitivos estudiados desde el paradigma de la Racionalidad Limitada, entendiendo a (al menos) algunos de ellos no como falencias de la razón humana sino como características que se vuelven positivas una vez que se las restituye a su escenario evolutivo original. La última sección propone algunas proyecciones de la Teoría Argumentativa de la Razón en el ámbito de la pedagogía y, especialmente, al momento de diseñar estrategias didácticas tanto al interior de la enseñanza de la filosofía como por fuera de ella.

\section{El optimismo epistémico de la racionalidad clásica y la Epistemología de la Virtud}

En tanto corriente epistemológica distintiva, la Epistemología de la Virtud ha estado atravesada desde su propio inicio en la última década del siglo pasado por la presencia de dos vertientes claramente diferenciadas y de escasa interacción mutua. La primera de dichas vertientes está repre- 
sentada por las reflexiones de Ernst Sosa y John Greco, fundamentalmente, en relación con el problema del confiabilismo, y tiene como uno de sus objetivos fundamentales responder al problema del escepticismo ${ }^{1}$. La segunda vertiente que se abre, en forma simultánea, dentro de dicha corriente está representada por un conjunto sumamente diverso y creciente de reflexiones de tipo normativo en relación con las vías y estrategias para generar y/o volvernos agentes epistémicos ideales ${ }^{2}$. Tal es la divergencia entre ambas vertientes, que la segunda de ellas no sólo no reconoce a Sosa y Greco (ni a A. Goldman) como antecedentes relevantes para sus propias exploraciones, sino que aún algunos de sus defensores consideran el problema del escepticismo como un problema de segundo orden. El punto de partida explícito de esta vertiente, por el contrario, se halla en las reflexiones iniciadas, a mediados del siglo pasado, por el modelo de las Éticas de la Virtud, y, en función de ello, tiende a interpretar las virtudes epistémicas más como rasgos del carácter que como instrumentos para alcanzar la verdad (como hacían Sosa y Greco) ${ }^{3}$.

Pero, ¿qué son las virtudes epistémicas y qué las diferencias de las virtudes de tipo ético o moral? En términos lo suficientemente generales como para cubrir el amplio espectro de virtudes analizadas por los autores (y recurriendo a una formulación de inspiración aristotélica), se puede definir a las virtudes epistémicas como disposiciones estables del carácter que permiten al sujeto realizar prácticas vinculadas con el conocimiento en forma ideal, o, alternativamente, las disposiciones del carácter necesarias para llevar a cabo una investigación honesta, cuidadosa, sensible al detalle, profunda, perseverante, reflexiva, cautelosa, etc. Lo que define, entonces, a las virtudes epistemológicas y las diferencias de las virtudes éticas es el hecho de que se relacionan específicamente con el problema del conocimiento y no con el problema de la relación con los otros (no al menos directamente). Y esto determina, a su turno, el espectro de las virtudes que serán objeto de estudio por los epistemólogos: coraje epistémico, humildad epistémica, apertura mental, rigor epistémico, etc.

Las raíces clásicas de esta concepción de lo que debería ser un ejercicio óptimo de la racionalidad se hacen evidentes no solo en cuanto a la concepción idealizada y optimista que los epistemólogos de la virtud poseen de la capacidades racionales humanas, sino del concepto mismo de virtudes epistémicas - concepto que se halla modelado sobre el tratamiento aristotélico de las virtudes éticas y dianoéticas (y que hereda, por otra parte, uno de los problemas centrales que aquejan al cognitivismo moderado del abordaje aristotélico, a saber: el problema de la akrasia $\left.{ }^{4}\right)$ Esta matriz aristotélica que se halla en las bases del programa de la Epis- 
temología de la Virtud ha demostrado ser sumamente fructífera para organizar la investigación sobre las virtudes intelectuales, no solo en lo relativo a la definición y conceptualización de las distintas posibles virtudes que caracterizan al ejercicio racional óptimo, sino también en cuanto a la detección de los posibles obstáculos para adquirir dichas virtudes, y en cuanto a la reflexión sobre las posibles vías de acceso a las mismas.

Desde el plano específico de la pedagogía, esto ha conducido a esbozar estrategias concretas que permitirían al alumno alcanzar objetivos tales como llevar adelante una investigación sistemática y rigurosa, ejercitar una defensa racional y sólida de sus propias conclusiones aún frente a un auditorio adverso, o emprender un diálogo/investigación con una mente abierta a opiniones completamente opuestas a la propia, obligándose a sí mismo a respetar las reglas de la justicia, la tolerancia y la paciencia con el interlocutor. Los análisis detenidos y profundos que los defensores de la Epistemología de la virtud han ofrecido tanto de las virtudes epistémicas aisladas, como de los agentes epistémicos virtuosos, han representado, en este sentido, el aporte fundamental y más innovador de dicha corriente al ámbito de la educación, en la medida en que ofrecen un marco integrado, y al mismo tiempo flexible y dinámico, para construir prácticas ideales de conocimiento, encarnadas en un ideal de sujeto cognoscente racional, crítico y autocrítico.

Desde los primeros pasos que diera Zagzebski hace tres décadas, abriendo con ello un terreno absolutamente nuevo de exploración teórica, la Epistemología de la Virtud ha producido un corpus sorprendentemente sólido de herramientas pedagógicas destinadas no solo a colaborar con la adquisición de virtudes epistémicas en el plano individual, sino, a largo plazo, a colaborar con lo que algunos de los epistemólogos más optimistas consideran una verdadera transformación del mundo a partir del desarrollo de dichas virtudes ${ }^{5}$. Es precisamente en la dimensión esencialmente práctica que asume, de esta forma, la epistemología realizada desde la perspectiva aretaica, abogando al mismo tiempo por el abandono de los abstractos problemas gnoseológicos tradicionales, en donde radica, precisamente, la razón más fuerte de su atractivo, tanto para quienes se dedican a la investigación como para quienes se dedican a la enseñanza, o a la reflexión sobre la misma.

Ahora bien: ¿cuán realista es el ideal epistémico defendido por la Epistemología de la Virtud? ¿Cuán asequibles son las virtudes epistémicas defendidas por estos autores y cuán superables son los obstáculos cuya existencia ellos mismos reconocen? ¿Es, a fin de cuentas, un programa de investigación viable? En las secciones subsiguiente se sugerirá que el pa- 
radigma de la racionalidad limitada y, más específicamente, la Teoría Argumentativa de la Razón ofrecen razones para arrojar serias dudas sobre el optimismo que caracteriza a la Epistemología de la Virtud en cuanto a las capacidades naturales del sujeto para alcanzar (o al menos acercarse) al ideal de sujeto epistémico defendido por dicha corriente. A pesar de ello, se sugerirá que la variante específica la Teoría Argumentativa de la Razón abre algunos senderos que, contra el pesimismo que atraviesa en forma sistemática al paradigma de la Racionalidad Limitada, permite pensar en reestructuraciones efectivas de la Epistemología de la Virtud que dejen atrás el optimismo ilustrado propio de las versiones originales de dichas corrientes.

\section{El pesimismo epistémico del paradigma de la Racionalidad Limitada}

En tanto modelo general y programático, el paradigma de la Racionalidad Limitada (Bounded Rationality) encuentra sus orígenes en las reflexiones tempranas de Herbert Simon (1955), en torno a las limitaciones que estructuran en forma inevitable a la racionalidad humana. El núcleo de dicho paradigma, en términos generales, consiste en la idea de que la racionalidad humana se encuentra limitada en sus operaciones por una serie de factores (tiempo, computabilidad del problema, limitaciones epistémicas, etc.) que vuelven a su desempeño necesariamente subóptimo. La razón humana no opera, en otras palabras, como una máquina inferencial perfecta y libre de errores, sino que, por el contrario, se halla atravesada por serias limitaciones en sus operaciones que ponen en cuestión el carácter ideal que la tradición clásica le había asignado. Lejos de representar una perogrullada, el hecho de que dicha idea haya perdido parte de su carácter controversial y contra-intuitivo en el ámbito académico, cabe remarcarlo, se debe fundamentalmente al impacto que tuvo dicho paradigma desde sus inicios en el ámbito de las ciencias sociales y en ciertos sectores de las disciplinas humanísticas. Pero es preciso tener en cuenta que la concepción limitada de la racionalidad representaba un quiebre decisivo con una tradición que había sido virtualmente hegemónica desde la antigüedad clásica hasta la década de 1950, y que partía del presupuesto de la absoluta racionalidad de los procesos inferenciales que subyacían a cada decisión humana.

Los desarrollos más importantes y sistemáticos de dicho paradigma, sin embargo, comenzaron a darse de la mano de las investigaciones desarrolladas en torno a los sesgos cognitivos por Daniel Kahneman y 
Amos Tversky en la década de 1970 (Kahneman \& Tversky, 1979; Tversky \& Kahneman, 1981), investigaciones desarrolladas fundamentalmente en contra de la Teoría de la Elección Racional (Rational Choice Theory), que es la teoría que, en el ámbito de la economía, encarnaba en ese momento la expresión más paradigmática de los presupuestos racionalistas de la tradición clásica. ¿Qué es lo que planteaba la teoría de los sesgos cognitivos? En líneas generales, que la razón humana se halla completamente viciada por sesgos de tipo cognitivo, es decir, tendencias espontáneas, inconscientes e intuitivas a procesar la información proveniente del entorno recurriendo a inferencias que nada tienen que ver con el modelo de racionalidad propio de la lógica clásica. El repertorio de sesgos estudiado por ambos autores se hizo cada vez mayor y más profundo, llegando a integrar tendencias como el 'sesgo de disponibilidad' (la tendencia a tomar en cuenta, en las decisiones, únicamente la información que está a la mano, fácilmente disponible, en lugar de buscar la información más relevante), el 'sesgo de falso consenso' (la tendencia a suponer que lo que uno cree o valora se halla más extendido entre la población que lo que realmente está), o el 'sesgo de confirmación' (la tendencia a prestar atención únicamente a la información que confirma las propias creencias y desestimar la información que las contradice).

Al término de décadas de investigación en torno a dichas tendencias, las conclusiones a las que llegaron quienes, junto con Kahneman y Tvers$\mathrm{ky}$, se dedicaron a estudiar estos sesgos, fueron sumamente negativas en relación con la capacidad humana de poner en juego realizar un ejercicio crítico, auto-crítico y eficiente de la racionalidad, conclusiones que parecen contradecir abiertamente las expectativas prácticas que atraviesan a las reflexiones desarrolladas en el seno de la Epistemología de la Virtud, y que parecen condenar al limbo de la pura ensoñación idealista del pedagogo la posibilidad de diseñar estrategias que permitan desarrollar en forma óptima las virtudes epistémicas. $Y$ es precisamente como alternativa a este escenario de pesimismo epistémico al que parece conducir en forma ineluctable el paradigma de la Racionalidad Limitada, que surgirá, a principios de la década pasada, el modelo de la Teoría Argumentativa de la Razón.

\section{La Teoría Argumentativa de la Razón: El surgimiento de la Razón y el paisaje evolutivo}

El estudio sistemático de los sesgos cognitivos evidenciaba que el modelo de racionalidad tradicional (defendido en la economía clásica, como se- 
ñalaba por la Teoría de la Acción Racional) no representaba en absoluto la dinámica real de la toma de decisiones: tanto al momento de consumir mercancías, productos o servicios, como al momento de tomar decisiones de tipo político o personal, o al enfrentar situaciones vinculadas con las relaciones de pareja o la propia carrera profesional, las decisiones que se toman son, en su inmensa mayoría, el resultado de procesos inferenciales inconscientes, sesgados y, en la mayor parte de los casos, deficientes desde un punto de vista lógico. Una lectura rápida de algunos de estos sesgos, así como de la frecuencia con la que se ponen en juego en forma cotidiana en las decisiones, no puede dejar de llevar a preguntarse, curiosamente, cómo es posible que, en tanto especie, se haya podido llegar tan lejos (en términos de supervivencia) con un vehículo tan mal construido.

Este interrogante, sobre el que han escrito durante las últimas décadas autores como Stanovich y West (2000, 2008), Evans (2008), Evans y Stanovich (2013), Bargh y Chartrand (1999), entre otros, es precisamente el que opera como disparador de la Teoría Argumentativa de la Razón: ¿Es realmente concebible que una capacidad tan deficiente como esta haya surgido como producto de la selección natural, considerando las serias deficiencias que se le atribuye desde la teoría de los sesgos cognitivos y desde otros sistemas de procesamiento dual? Desde la perspectiva del evolucionismo clásico, una modificación fenotípica en un organismo se vuelve objeto de la selección natural solo si la misma es adaptativa, es decir: si le confiere a dicho organismo ( $y$ a su descendencia) una ventaja adaptativa en un determinado escenario evolutivo. Si se admite, como hacen tanto Simon como Kahneman y Tversky, que el modelo teórico de la evolución por selección natural es el mejor modelo de explicación que ha sido propuesto hasta el momento para explicar no solo el surgimiento, mutación y extinción de las especies, sino también el surgimiento, mutación y extinción de los rasgos fenotípicos, la imagen que la teoría de los sesgos cognitivos devuelve de la razón humana parece ser marcadamente no adaptativa. ¿Cómo explicar, entonces, su surgimiento y proliferación? Es allí, precisamente, en donde la TAR introduce su giro argumentativo más interesante en relación con el paradigma de la Racionalidad Limitada, sin por ello desacoplarse del mismo ${ }^{6}$ de acuerdo con Mercier y Sperber (2019), la razón humana es marcadamente deficiente, en efecto, pero solo si se parte del presupuesto de que el objetivo de la razón es alcanzar la verdad, o alcanzar un conocimiento cada vez más adecuado del mundo (tal como supusieron que era el caso, desde la antigüedad en adelante, tanto la filosofía como la psicología clásica).

Pero ¿es ese realmente el caso? ¿Es realmente cierto que cuando se argumenta lo que se intenta hacer es buscar la verdad, conocer mejor 
el mundo y la situación en la que uno se encuentra, simplemente para comprenderla o para poder tomar la mejor decisión posible? Mercier y Sperber (2019) sugieren que no: cuando se argumenta, cuando se exigen razones y se ofrecen las propias, lo que se está buscando, en la mayor parte de los casos, no es la verdad, sino persuadir al otro respecto de la verdad de la propia posición. Se encuentra aquí, de esta forma, una de las dos distinciones centrales que la TAR obliga a realizar para comprender los mecanismos operativos de la razón humana, a saber: la distinción entre los contextos naturales y artificiales de operación de la razón. Esta distinción es central por dos razones: en primer lugar, porque define el contexto específico de surgimiento de la razón: el diálogo con el otro. La razón, en este sentido, es un producto diseñado para el consumo públi$\mathrm{co}^{7}$ : un individuo aislado, que viviera solo en el medio de la selva y no tuviera contacto con otros individuos de su misma especie, jamás sentiría la necesidad de argumentar en favor de sus propias creencias, o siquiera de reflexionar acerca de las razones que lo llevan a hacer lo que hace.

Esto trae aparejado un segundo elemento que los autores se preocupan en enfatizar, y tiene que ver con el (no) lugar que asumen el conocimiento y la verdad en el desarrollo de la racionalidad: en la mayor parte de los contextos naturales de argumentación (desde el punto de vista del escenario evolutivo), como ya se ha mencionado, la búsqueda de la verdad no es en absoluto el objetivo que se persigue; lo que se persigue es meramente la persuasión, en general por cualquier medio $-\mathrm{y}$ a casi cualquier costo. La verdad, a fin de cuentas, tiene tanto valor en el ámbito de la supervivencia como la tipografía elegida por el editor al momento de comprar un libro. En el ámbito de la interacción humana espontánea, lo que prima, al menos desde la perspectiva sugerida por los autores, no es la verdad, sino los efectos de un determinado discurso en el (o los) interlocutor(es) de turno. Y esto se debe fundamentalmente a que el diálogo con el otro en el que surge como nicho evolutivo la capacidad de argumentar no es un diálogo objetivo, frío y especulativo, ni se trata de un intercambio neutro o carente de consecuencias: en el paisaje evolutivo en el que toma forma gradualmente la razón humana, convencer o no convencer al otro puede significar la diferencia entre tener acceso a ciertos bienes, escenarios o situaciones, o no tenerlo; llevado a un extremo, el dominio de dicha capacidad puede significar la diferencia entre la supervivencia o la extinción. La razón es, considerada desde esta perspectiva, un producto social y agonístico: es hija del conflicto, de la lucha por el acceso a ciertos bienes y ventajas — ya sean simbólicas o materiales. 
Lejos de reducirse a una recaída en los horizontes pragmáticos de la sofística clásica, la perspectiva abordada por esta teoría aspira a ayudar a comprender la razón humana como un fenómeno histórico, como un producto marcado por el escenario evolutivo en el que surgió, y cuyas marcas están aún presentes en su estructura actual. El desplazamiento que esto opera respecto de la concepción clásica de la razón (sobre la que se funda gran parte de las reflexiones y proyecciones en torno a la didáctica de la filosofía) es evidente: mientras que la concepción tradicional interpreta a la razón como una herramienta para alcanzar la verdad, el consenso objetivo, etc., la TAR ubica a la razón en el ámbito natural de la evolución humana, y postula los escenarios de búsqueda objetiva, neutral y disciplinada de la verdad como escenarios artificiales o directamente antinaturales.

Como se señalaba anteriormente, desde el paradigma evolucionista

212 del que parten los autores, un determinado rasgo fenotípico (en este caso la razón humana) es adaptativo en la medida en que cumple la función para la que fue 'seleccionada', y puede operar en forma subóptima cuando es puesta a funcionar en escenarios alternativos: así como no se puede esperar que la mano de un chimpancé o de un bonobo sea eficiente para tocar el clarinete, tampoco se debería esperar que la razón humana sea eficiente en la búsqueda objetiva de la verdad — sencillamente por el hecho de que no es esa la función para la que fue seleccionada. Cuando se extrae a la razón humana del horizonte de la argumentación agonística en el cual evolucionó y se la pone a trabajar en otro escenario, es lógico que su desempeño sea deficiente, y es lógico que se halle atravesada por sesgos completamente contraproducentes. Nada de esto implica, desde ya, que todas las operaciones de la razón se hallen guiadas ineluctablemente por la búsqueda de la persuasión, ni que los seres humanos sean incapaces de planificar y secuenciar argumentos sólidos en favor de sus propias creencias. Todo lo que la TAR afirma es que cuando sucede lo contrario, es decir, cuando los sujetos privilegian la búsqueda de la verdad por sobre la persuasión del interlocutor, o cuando diseñan y planifican largas argumentaciones secuenciadas y estructuradas lógicamente, se está ante situaciones que difieren del escenario evolutivo original de la razón.

\section{La reconsideración de los sesgos cognitivos}

Decía al principio que la TAR introduce un quiebre al interior del paradigma de la Racionalidad Limitada, pero sin abandonar el horizonte general definido por dicho paradigma. Para la TAR, la razón se halla, en efecto, 
atravesada por sesgos cognitivos que permean sus operaciones, y su desempeño se halla indudablemente atado a limitaciones como el tiempo o la computabilidad de las alternativas de decisión. Las críticas de Mercier y Sperber al optimismo Ilustrado de corrientes como la Epistemología de la Virtud son, en este sentido, tan fuertes como las de Kahneman (2012), Evans y Stanovich (2013), Gigerenzer (2008) o Nickerson (1998).

Sin embargo, la consideración del paisaje evolutivo en el que surge la razón humana conduce a los autores a realizar una reconsideración de la eficiencia argumentativa de la razón, temperando con ello, al menos en ciertos aspectos, el pesimismo epistémico propio del paradigma de la Racionalidad Limitada. Pero ¿̇a qué se debe esta reconsideración? Se afirmaba anteriormente que, en sus operaciones cotidianas, y como ha insistido hasta el hartazgo este último paradigma, la razón humana no es particularmente eficiente al momento de producir argumentos sólidos y sistemáticos, lo cual se debe, de acuerdo con Mercier y Sperber (2019), al hecho de que esa no es, precisamente, la función para la cual fue seleccionada por el proceso evolutivo. La contracara de este argumento, sin embargo, ha sido virtualmente desatendida, y consiste en el hecho de que, como sugiere una batería de experimentos provenientes de la psicología experimental reseñados por los autores, la razón sí es sumamente eficiente cuando hace lo que está diseñada para hacer, a saber: argumentar en un contexto agonístico.

Los contextos de discusión informal que representan más adecuadamente el escenario evolutivo de la razón representan, hasta cierto punto, la antítesis de los tribunales de justicia, o de los escenarios de argumentación lógica, exhaustiva y sistemática que la concepción tradicional de la racionalidad ha utilizado como criterio para evaluar la eficiencia de la razón humana.

A diferencia de las argumentaciones secuenciadas, planificadas y articuladas de los tratados de filosofía o de los alegatos jurídicos, la dinámica que se establece en forma espontánea en los contextos informales de discusión es esencialmente interactivo, lo cual implica que los participantes intercambian una sucesión de argumentos breves y, frecuentemente, improvisados o, cuanto menos, adecuado a las circunstancias específicas no solo de esa discusión en particular, sino también del momento específico de la discusión. En escenarios de ese tipo, ser "vago" es una decisión comprensible y sensata, y esto por dos razones. La primera de ellas es que tener preparados de antemano extensos argumentos para cada una de las propias afirmaciones exigiría un trabajo cognitivo insostenible. La segunda razón es que probablemente representaría un trabajo innecesario, 
dado que, por un lado, lo más probable es que la mayoría de dichas afirmaciones no sean objetadas, y, por otro, porque la naturaleza dinámica del diálogo permite improvisar nuevas razones cuando se ha fallado en el intento de convencer al interlocutor.

Esta relocalización de la razón en el contexto evolutivo permite una relectura decisiva de los sesgos cognitivos que obliga a matizar algunas de las conclusiones más pesimistas (desde el punto de vista epistémico) a las que llegaban Kahneman, Tversky, Nickerson y demás, dado que permite pensar que al menos algunos de los sesgos cognitivos estudiados en forma exhaustiva desde el paradigma de la Racionalidad Limitada pueden no ser, en rigor, deficiencias de la razón, sino, más bien, características positivas. El extenso análisis que Mercier y Sperber realizan del sesgo de confirmación constituye un ejemplo paradigmático de la reinterpreta214 ción que proponen de estos sesgos, y permite vislumbrar las ventajas hermenéuticas del abordaje propuesto por los autores.

En efecto, el sesgo de confirmación (esto es, la tendencia inconsciente y espontánea a prestar atención únicamente a la información que confirma las propias creencias y a desestimar la que no lo hace), tradicionalmente considerado como una de las tendencias más nocivas desde el estudio clásico de Nickerson (1998), aparece desde la perspectiva de la TAR como una característica necesaria cuando se consideran los contextos informales de discusión y la función (evolutiva) esencialmente agonística y persuasiva de la razón humana. Las razones de ello son claras: cuando se quiere convencer a un interlocutor de aceptar una creencia propia como válida o verdadera, lo que se necesita hacer es encontrar razones que confirmen la propia posición, y no la suya, y toda información o argumento que pueda minar ese objetivo persuasivo se vuelve absolutamente irrelevante. Considerado desde esta perspectiva, entonces, el sesgo de confirmación exige ser comprendido no como una debilidad de la razón humana, sino como una fortaleza, en la medida en que le permite al sujeto buscar y desplegar en forma activa las razones que fundamentan las creencias que intenta imponer. Como señalan Mercier y Sperber (2017):

Los sesgos y la pereza de la razón no son falencias; son características que le permiten a la razón cumplir su función. Los individuos tienen una tendencia (sesgo) a encontrar razones que apoyen su propio punto de vista porque así es como pueden justificar sus acciones y convencer a los otros de compartir sus opiniones. Uno no puede justificarse a sí mismo presentando razones que refuten la propia justificación. Uno no puede convencer a otro de cambiar de idea dándole argumentos en contra de la misma o a favor de la idea que le quiere hacer abandonar. Y si la 
gente razona con pereza, esto se debe a que, en las interacciones típicas, esa es la forma más eficiente de proceder. En lugar de realizar el arduo trabajo de anticipar contra-argumentos, por lo general es más eficiente esperar a que el interlocutor lo haga (si es que lo hace) (p. 331).

A esto se suma, por último, una última distinción decisiva que proponen los autores para comprender la dinámica espontánea de la razón en contextos naturales, que es la distinción entre la eficacia de la razón al momento de producir argumentos y su eficacia para evaluar los argumentos propuestos por otros. De acuerdo con los autores, y nuevamente apoyándose en los resultados de un conjunto de investigaciones procedentes de la psicología experimental, las limitaciones de la razón humana al momento de producir argumentos no se replica al momento de evaluarlos: si al momento de la producción de argumentos se es vago, superficial, etc., y se está constantemente atravesado por sesgos (lo cual, considerado en sí mismo, no es, como ya se señaló, una falencia, sino algo esperable), al momento de evaluar los argumentos propuestos por los interlocutores se es mucho más eficaz, profundo y crítico. Como señalan Mercier y Sperber (2017): "Los individuos poseen la capacidad de razonar en forma objetiva, rechazando argumentos débiles y aceptando los que son sólidos, solo que no usan estas capacidades sobre las razones que ellos mismos ofrecen" (p. 235). Esto es, precisamente, lo que cabría esperar de una herramienta, como la capacidad humana de argumentar, nacida en un contexto agonístico, en el cual la energía volcada a la persuasión activa del interlocutor termina volcándose, cuando los roles se intercambian, en una energía defensiva, corporizada en el examen crítico de los argumentos del contrincante.

\section{Proyecciones pedagógicas de la Teoría Argumentativa de la Razón}

El aporte central de la TAR en relación con la problemática del optimismo epistémico que caracteriza a corrientes como la Epistemología de la Virtud, y con el pesimismo epistémico que caracteriza al paradigma de la Racionalidad Limitada, consiste, como he intentado mostrar, en sugerir que ciertos sesgos cognitivos deben ser interpretados, desde una perspectiva evolutiva, no como un obstáculo o una falencia, sino, por el contrario, como una ventaja cognitiva, o un aspecto positivo de la razón ("It's not a bug; it's a feature!”). Este giro respecto del paradigma de Kahneman y Tversky es decisivo, desde el punto de vista de la consistencia interna de 
la teoría, en la medida en que da respuesta al interrogante que se mencionaba que opera como disparador de las reflexiones que condujeron a formular la TAR, a saber: ¿Cómo es posible que un instrumento tan limitado en sus operaciones y atravesado por sesgos haya sido adaptativo y, en consecuencia, seleccionado naturalmente? La respuesta de Mercier y Sperber (2017), en relación con esta pregunta, es sencilla: el carácter adaptativo de la razón humana radica precisamente en (algunas de) aquellas características que el paradigma de la Racionalidad Limitada considera como falencias, pero que, cuando se las restituye al paisaje evolutivo correcto, se muestran como características positivas y beneficiosas para la función original por la que fue seleccionada la razón, a saber: argumentar para persuadir (y no para buscar la verdad o el conocimiento).

Este giro, sin embargo, es interesante por otra razón, esta vez de orden pragmático: en la medida en que opera como un correctivo frente al pesimismo epistémico propio del paradigma de la Racionalidad Limitada, permite dotar nuevamente de sentido al diseño de estrategias pedagógicas tendientes a mejorar las prácticas y hábitos epistémicos del sujeto -algo que virtualmente carecía de sentido si se partía del pesimismo aplastante de aquel paradigma. Esto no implica, desde ya, un retorno al optimismo propio de la Epistemología de la Virtud, el cual parte de una concepción Ilustrada cuasi-rousseauniana del sujeto, un sujeto que no estaría atravesado por sesgos cognitivos, morales o políticos, y que estaría guiado, al menos la mayor parte del tiempo, por el deseo del conocimiento y la verdad. Nada de esta confianza excesiva, ingenua y, hasta cierto punto voluntarista, en las capacidades racionales del hombre se verá restituido por la TAR. Lo que sí se abre es el desafío de pensar estrategias pedagógicas que partan del hecho dado de que se posee una determinada estructura cognitiva (atravesada por sesgos y guiada inconscientemente, la mayor parte del tiempo, por la necesidad de persuadir) que es el resultado de un proceso evolutivo, que puedan hacer uso precisamente de esas características, en lugar de ignorarlas, y ponerlas a funcionar en escenarios que, entre otras cosas, repliquen las características del paisaje evolutivo de la razón humana.

En este punto se abren fundamentalmente dos perspectivas (divergentes pero complementarias entre sí): una constructiva y otra destructiva. La perspectiva constructiva esboza, en cierta medida, las condiciones bajo las cuales una determinada estrategia didáctica puede ser efectiva en relación con el objetivo de estimular las capacidades argumentativas de los participantes - $\mathrm{o}$, cuanto menos, obliga a reflexionar acerca de dichas condiciones, en lugar de meramente suponer que cualquier estrategia di- 
dáctica es efectiva por el mero hecho de apelar al diálogo, a la autocrítica o a la búsqueda de razones.

El primer corolario que se deriva de las premisas propuestas por TAR es el hecho de que cuando se argumenta, la calidad y solidez de los argumentos dependerá del auditorio al que uno se vea enfrentado, de cuán contra-argumentativo sea el interlocutor: para producir buenos argumentos, se necesita de la presencia de un interlocutor crítico, que empuje a producir argumentos sólidos, convincentes. La mera consigna de defender una determinada posición en una exposición ante un grupo, por ejemplo, se vuelve completamente improductiva si el sujeto sabe de antemano que la posición expuesta no va a ser objetada y cuestionada abiertamente. La profundidad y solidez de los argumentos, en pocas palabras, estarán en función directa con la interacción crítica y activa con los interlocutores de turno ${ }^{8}$.

El segundo corolario, y he aquí lo fundamental desde el punto de vista constructivo, es que la única forma de superar o contrarrestar el efecto negativo de los sesgos cognitivos propios del razonamiento humano es poniéndolos a trabajar en nuestro favor, lo cual se puede lograr, básicamente, de dos maneras: estimulando el razonamiento proactivo y diseñando espacios de debate argumentativo conflictivo. ¿Qué se entiende por pensamiento proactivo? De acuerdo con Mercier y Sperber (2017), cuando los individuos razonan en aislamiento tienden en ocasiones, y dependiendo del escenario que saben que van a enfrentar al exponer sus convicciones, a emular un contexto argumentativo agonístico, anticipando un posible contexto dialógico e intentando buscar argumentos que confirmen su propia posición. El pensamiento proactivo es precisamente ese ejercicio de razonar, en forma aislada e individual, buscando argumentos en favor de las propias creencias, como si se estuviera discutiendo con otros, y anticipándose a sus objeciones y pensando de antemano respuestas a las mismas. Es claro, desde ya, que esto no es lo que se hace todo el tiempo, sino solo cuando se sabe o se sospecha que uno se va a ver enfrentado a una situación en la que se exigirá que se dé cuenta de las propias razones. La segunda estrategia sugerida por los autores para poner a trabajar los sesgos cognitivos a favor de uno mismo consiste en la construcción de espacios de debate argumentativo —espacios que tienden, por su propia dinámica, y siempre que se hallen organizados por un mediador competente, a transformar el sesgo de confirmación en una herramienta de despliegue lógico de las razones que apoyan la posición de cada uno de los participantes. 
Pero la TAR contribuye a comprender no solo qué tipo de escenarios y estrategias son realmente efectivos para estimular la racionalidad argumentativa, sino también cuáles no lo son - y es ahí donde entra en juego lo que se puede llamar la perspectiva negativa. En primer lugar, si el sesgo de confirmación es una característica efectiva de la razón humana, (y si, adicionalmente, existen razones que permiten interpretarlo como una característica positiva de la razón — al menos dentro de ciertos contextos), entonces toda estrategia didáctica construida sobre la idea de un pensamiento autocrítico parece estar destinada al fracaso en vistas de la arquitectura cognitiva que se ha heredado de los antepasados: pocos sujetos, de acuerdo con estas premisas, se cuestionan sus propias creencias en forma espontánea. En general, para que se sometan a revisión las convicciones, es necesario que se dé alguno de los siguientes escenarios: que las mismas entren en conflicto con las creencias de otro sujeto, o con alguna característica del escenario en el que se está inmerso, ya sea porque alguien lo obliga a uno a dar cuenta de ellas, o porque chocan de alguna manera con la realidad, o bien, por último, porque ya no son efectivas, es decir: porque ya no producen los efectos que producían hasta el momento 9 . En segundo lugar, del hecho de que el razonamiento humano sea particularmente eficaz en contextos argumentativos, no se deriva que cualquier estrategia de discusión grupal pueda ser efectiva en sí misma. Como afirman Mercier y Sperber (2017):

Cuando los participantes poseen desde el inicio convicciones claramente alineadas entre sí, ello conduce a la polarización. Cuando los sujetos comienzan la discusión con ideas que están en conflicto entre sí y no poseen un objetivo compartido, ello tiende a exacerbar las diferencias. La discusión grupal es típicamente beneficiosa cuando los participantes tienen ideas diferentes y un objetivo compartido (p. 334).

Consideradas en forma conjunta, las indicaciones positivas y las restricciones mencionadas ponen sobre el escenario la necesidad de atender a la especificidad de los escenarios artificiales de argumentación diseñados con fines pedagógicos, y la necesidad de comprender la dinámica espontánea de las propias capacidades argumentativas. La razón humana, a fin de cuentas, no es un módulo de resolución general y universal, sino un módulo (o un conjunto de ellos) especializado y surgido en el seno de un determinado escenario evolutivo. No tomar en consideración esas características al momento de diseñar estrategias didácticas solo puede conducir al diseño de estrategias pedagógicas ingenuas e inefectivas y, al final del camino, al fracaso de los sueños de la Ilustración a los que los epistemólogos de la virtud se siguen aferrando. 


\section{Conclusiones}

Cabe prestar atención, a modo de consideración final, a un último punto que no es en absoluto exclusivo de la TAR sino que atañe a la didáctica de la filosofía considerada globalmente, y es el de la complementación entre el marco teórico y el apoyo empírico: TAR ofrece no solo una marco teórico general (el de la racionalidad como producto de la selección natural), sino también un cúmulo de estudios provenientes del ámbito de la psicología experimental que corroboran, al menos provisoriamente, las predicciones de la teoría. El diseño de estrategias pedagógicas basadas únicamente en presupuestos teóricos acerca de la racionalidad humana, pero sin ningún tipo de investigación empírica que respalde su posible efectividad, parece condenado a transitar el camino seguido, a modo de ejemplo, por una estrategia didáctica como el brainstorming, una estrategia cuya marcada ineficacia para estimular el debate argumentativo y para conducir a la búsqueda de nuevas soluciones parece haber sido ya claramente demostrado ${ }^{10}$. Desestimar los aportes de otras disciplinas en el diseño de estrategias didácticas, en este sentido, no parece ya ser una vía recomendable, y esto se vuelve particularmente decisivo en relación con los aportes de la psicología y, fundamentalmente, de la psicología experimental: ¿cuál es la mejor manera de garantizar el compromiso de un participante con el debate después de que su posición ha sido abiertamente puesta en cuestión? ¿Qué tipo de actitudes suelen adoptar los sujetos cuando se ven enfrentados a escenarios de debate agresivo? ¿Qué ventajas efectivas ofrece, a este respecto, el hecho de garantizar un espacio de debate respetuoso y tolerante? ¿Cuáles son los modos en los que los sujetos suelen resolver los casos de disonancia cognitiva en escenarios de debate grupal, en donde se exige una respuesta rápida?

Este tipo de interrogantes es esencial para diseñar estrategias efectivas, y solo puede ser respondido en forma satisfactoria por un abordaje que ofrezca, en primer lugar, un marco teórico sólido y articulado con el resto de las disciplinas (humanísticas y no humanísticas) en cuanto al concepto de racionalidad humana, $y$, en segundo lugar, un apoyo empírico considerable, renovado y dinámico. La TAR cumple con ambos requisitos. No es la única alternativa disponible, desde ya. Pero parece ser una plataforma sólida, plausible, y sumamente flexible para repensar nuestras prácticas docentes y las estrategias didácticas que implementamos institucionalmente. 
Didactical projections of the argumentative theory of reasoning

\section{Notas}

1 Si bien se suele considerar a Sosa 1980 como la piedra de toque de esta primera vertiente de la Epistemología de la Virtud, Sosa (2011) y Greco (2010) representan dos vías de ingreso más sistemáticas y accesibles a los lineamientos generales de la misma.

2 Dentro de esta segunda vertiente, Zagzebski (1996) representa, en buena medida, el texto fundacional, tanto desde un punto de vista metodológico como temático. Roberts y Wood (2007) y Baehr (2011) constituyen los dos abordajes sistemáticos de referencia más recientes, además de la compilación realizada por Fairweather y Zagzebski (2001).

3 Roberts y Wood (2007) ofrecen una definición sintética pero comprehensiva y programática de esta segunda vertiente de la Epistemología de la Virtud: "Virtue epistemology, as we understand it, explores dispositional properties of persons that bear on the acquisition, maintenance, transmission, or application of knowledge and allied epistemic goods such as truth, justification, warrant, coherence and interpretative fineness. Personal traits that regularly promote such goods are virtues, and ones that impede or undermine them are vices. Relevant dispositional properties are of at least two kinds. [...] It is an a posteriori normative conceptual discipline; it aims to describe knowers at their best, so it describes an ideal" (p. 257).

4 Cf. al respecto Battaly (2014).

5 Roberts y Wood (2007) representa un ejemplo paradigmático de este énfasis.

6 El núcleo central de la TAR se encuentra desarrollado en Mercier y Sperber (2017 y 2019). Si bien entre ambos textos se produce un distanciamiento decisivo respecto de los modelos duales de explicación de la acción humana y un viraje hacia modelos como el intuicionismo social (tal como el defendido en Haidt, 2001), no existen diferencias esenciales respecto de la interpretación que los autores proponen de los ejes comentados en estas páginas. Proyecciones adicionales de la teoría se encuentran en Mercier (2011 y 2019) y Mercier y Heintz (2014). Las traducciones de Mercier y Sperber (2017) son, en todos los casos, del autor del presente artículo.

7 No es casual, en este sentido, que Mercier y Sperber (2017) vinculen explícitamente, en la exposición más reciente de la TAR, el concepto de razón con el de 'reputación': "La reputación de una persona es, en buena medida, el efecto continuo de una conversación desplegada en el tiempo y en el espacio social acerca de las razones de esa persona. $\mathrm{Al}$ dar nuestras razones, aspiramos a participar en la conversación acerca de nosotros y defender nuestra reputación. [...] Dar razones para justificar las propias acciones y reaccionar ante las razones que ofrecen los demás es, en primer y fundamental lugar, a forma de establecer reputaciones y de coordinar expectativas" (pp. 142-143).

8 Lo que se encuentra en juego en este señalamiento no es otra cosa que la necesidad epistémica de transitar lógicamente cada camino hasta sus últimas consecuencias, lo cual remite ineludiblemente a la idea popperiana de dejar que nuestras hipótesis mueran en lugar de nosotros, y representa, de alguna manera, una imagen especular de la premisa más importante de la metodología socrática, a saber, obligar al interlocutor a desplegar la idea al máximo, hasta encontrar su límite, y, eventualmente su contradicción interna o su conflicto con otras ideas defendidas por el sujeto. Como tanto Sócrates, Popper, y Mercier y Sperber comprendieron, esto es algo que solo puede acaecer en el seno de la dialéctica propia del conflicto argumentativo (ya sea real o a través de la dinámica de la empresa científico-filosófica). Finalmente, como remarcan Mercier y Sperber (2017), esto da por tierra con el mito del 'genio solitario': los grandes logros de la razón no han sido nunca el producto de una mente 
individual, sino un producto colectivo, fruto de la interacción, siempre conflictiva, entre varios individuos a lo largo de muchas generaciones (pp. 315-327).

9 Esto explica, incidentalmente, el carácter excepcional de la capacidad de autocrítica en las relaciones marcadamente jerárquicas, de parte de quien se encuentra en la posición de poder. Como señalan Mercier y Sperber (2019), "muchas de nuestras creencias son propensas a permanecer sin ser desafiadas porque solo son relevantes para nosotros mismos y no las compartimos, o porque son objeto de controversia únicamente con las personas con las que interactuamos, o porque tenemos la autoridad suficiente para afirmarlas" (p. 26).

10 Cf., a modo de ejemplo, Diehl y Stroebe (1987) y Mullen, Johnson y Salas (1991).

\section{Bibliografía}

BAEHR, Jonathan

2011 The Inquiring Mind: On Intellectual Virtues and Virtue Epistemology. Oxford: Oxford University Press.

BARGH, John \& CHARTRAND, Tanya

1999 The unbearable automaticity of being. American Psychologist, 54(7), 462479. https://doi.org/10.1037/0003-066X.54.7.462

BATTALY, $\mathrm{H}$.

2014 Acquiring Epistemic Virtue: Emotions, Situations, and Education. En A. Fairweather, O. Flanagan (Eds.), Naturalizing Epistemic Virtue (pp. 175196). Cambridge: Cambridge University Press.

DIEHL, Michael \& STROEBE, Wolfgang

1987 Productivity loss in brainstorming groups: Toward the solution of a riddle. Journal of Personality and Social Psychology, 53(3), 497-509. https://doi. org/10.1037/0022-3514.53.3.497

EVANS, Jonathan

2008 Dual-processing accounts of reasoning, judgment, and social cognition. Annual Review of Psychology, 59, 255-278. https://doi.org/10.1146/annurev. psych.59.103006.093629

EVANS, Jonathan \& STANOVICH, Keith

2013 Dual-Process Theories of Higher Cognition: Advancing the Debate. Perspectives on Psychological Science, 8(3), 223-241. https://doi. org/10.1177/1745691612460685

FAIRWEATHER, Abrol \& ZAGZEBSKI, Linda (Eds.)

2001 Virtue Epistemology: Essays on Epistemic Virtue and Responsibility. Oxford: Oxford University Press.

GIGERENZER, G.

2008 Moral intuition = Fast and frugal heuristics? En W. Sinnott-Armstrong (Ed.), Moral Psychology. 2. The Cognitive Science of Morality: Intuition and Diversity (pp. 1-27). MIT Press.

GRECO, John

2010 Achieving knowledge: A virtue-theoretic account of epistemic normativity. Cambridge: Cambridge University Press. 
Didactical projections of the argumentative theory of reasoning

HAIDT, Jonathan

2001 The emotional dog and its rational tail: A social intuitionist approach to moral judgment. Psychological Review, 108(4), 814-834. https://doi. org/10.1037//0033-295X.108.4.814

KAHNEMAN, Daniel

2012 Pensar rápido, pensar despacio. Madrid: Debate.

KAHNEMAN, Daniel \& TVERSKY, Amos

1979 Prospect Theory: An analysis of decision under risk. Econometrica, 47(2), 263-291. https://doi.org/10.2307/1914185

MERCIER, Hugo

2011 What good is moral reasoning? Mind \& Society, 10(2), 131-148. https://doi. org/10.1007/s11299-011-0085-6

MERCIER, Hugo \& HEINTZ, Christophe

2014 Scientists' Argumentative Reasoning. Topoi, 33(2), 513-524. https://doi. org/10.1007/s11245-013-9217-4

MERCIER, Hugo \& SPERBER, Dan

2017 The Enigma of Reason: A New Theory of Human Understanding. Cambridge: Harvard University Press.

2019 ¿Por qué razonan los humanos? Argumentos para una Teoría Argumentativa (C. McDonnell \& J. M. Vivas, Trads.). Cuadernos Filosóficos / Segunda Época, 15. https://doi.org/10.35305/cf2.vi15.54

MULLEN, Brian, JOHNSON, Craig \& SALAS, Eduardo

1991 Productivity loss in brainstorming groups: A meta-analytic integration. Basic and Applied Social Psychology, 12(1), 3-23. https://doi.org/10.1207/ s15324834basp1201_1

NICKERSON, Raymond

1998 Confirmation bias: A ubiquitous phenomenon in many guises. Review of General Psychology, 2(2), 175-220. https://doi.org/10.1037/1089-2680.2.2.175

ROBERTS, Robert \& WOOD, Jay

2007 Intellectual virtues: An essay in regulative epistemology. Oxford: Oxford University Press.

SIMON, Herbert

1955 A Behavioral Model of Rational Choice. The Quarterly Journal of Economics, 69(1), 99-118. https://doi.org/10.2307/1884852

SOSA, Ernest

1980 The Raft and the Pyramid: Coherence versus Foundations in the Theory of Knowledge. Midwest Studies In Philosophy, 5(1), 3-26. https://doi. org/10.1111/j.1475-4975.1980.tb00394.x

2011 Knowing Full Well. Princeton: Princeton University Press.

STANOVICH, Keith \& WEST, Richard

2000 Individual differences in reasoning: Implications for the rationality debate? Behavioral and Brain Sciences, 23(5), 645-665. https://doi.org/10.1017/ S0140525X00003435

STANOVICH, Keith \& WEST, Richard

2008 On the relative independence of thinking biases and cognitive ability. Journal of Personality and Social Psychology, 94(4), 672-695. https://doi. org/10.1037/0022-3514.94.4.672 
TVERSKY, Amos \& KAHNEMAN, Daniel

1981 The framing of decisions and the psychology of choice. Science, 211(4481), 453-458. https://doi.org/10.1126/science.7455683.

ZAGZEBSKI, Linda

1996 Virtues of the mind: An inquiry into the nature of virtue and the ethical foundations of knowledge. Cambridge: Cambridge University Press.

Fecha de recepción de documento: 15 de julio de 2020

Fecha de revisión de documento: 15 de septiembre de 2020

Fecha de aprobación de documento: 15 de octubre de 2020

Fecha de publicación de documento: 15 de enero de 2021 